Instituto Internacional de Investigación y Desarrollo Tecnológico Educativo INDTEC, C.A.

DOI: https://doi.org/10.29394/scientific.issn.2542-2987.2017.2.6.4.74-91

OAI-PMH: http://www.indteca.com/ojs/index.php/Revista Scientific/oai

\title{
Estrategias Didácticas para mejorar la Lectura y la Escritura
}

Autores: Elide del Rosario Castellanos Santiago Universidad Nacional Experimental "Rafael María Baralt", UNERMB elidecastellanos255@hotmail.com

Trujillo, Venezuela Javier José Castro Capitillo Universidad Nacional Experimental "Rafael María Baralt", UNERMB jccapitillo@hotmail.com

Trujillo, Venezuela

\section{Resumen}

La importancia del presente artículo radica en que intenta reflexionar acerca degenerar orientaciones teóricas sobre estrategias didácticas para mejorar la lectoescritura en los estudiantes de la escuela Mercedes Díaz de la parroquia Sabana de Mendoza, municipio Sucre, estado Trujillo. Metodológicamente, la investigación fue de carácter documental, descriptiva, sustentada con los postulados de Lev Vygotsky (1993), Piaget (1978) y Emilia Ferreiro (2000), Ortiz, (2008) Flower y Hayes (2009). Los resultados obtenidos evidenciaron la participación efectiva por parte de los docentes y el compromiso adquirido a través del manejo y aplicación de las estrategias didácticas de lectura y escritura que pueden favorecer el aprendizaje del sujeto que aprende.

Palabras clave: lectura oral; escritura manuscrita; método de enseñanza. 


\section{Didactic Strategies to Improve Reading and Writing}

\section{Abstract}

The importance of the present article is that it tries to reflect on generating theoretical orientations on didactic strategies to improve the literacy in the students of the school Mercedes Diaz of the parish Sabana de Mendoza, municipality Sucre, Trujillo state. Methodologically, the research was documentary, descriptive, supported by the postulates of Lev Vygotsky (1993), Piaget (1978) and Emilia Ferreiro (2000), Ortiz, (2008) Flower and Hayes (2009). The obtained results evidenced the effective participation by the teachers and the commitment acquired through the management and application of didactic strategies of reading and writing that can favor the learning of the subject that learns.

Keywords: oral reading; handwriting; teaching method.

Date Received: 26-05-2017 


\section{Introducción}

Hablar de las estrategias didácticas es hacer mención a los procedimientos que el docente utiliza en forma consecuente, vigilada y deliberada como instrumentos flexibles para aprender significativamente y solucionar problemas. Al respecto, Nogales (2000a), señala "son un conjunto de estrategias educativas, métodos, quehaceres, entre otros, que utiliza el maestro en el aula para explicar, hacer, comprender, motivar, estimular y orientar los procesos de enseñanza y aprendizaje" (pág. 21). De hecho, Nogales (2000b), muestra que para "poder desarrollar de manera correcta una intervención educativa se necesitan muchas cosas, entre ellas se aplica un conjunto de estrategias que facilitan la labor docente" (pág. 29).

De lo anterior se puede señalar, que todo docente debe poseer en su planificación particular de trabajo un sin fin de estrategias que motiven al estudiante a aprender de manera práctica, donde sea él su propio constructor de su aprendizaje. Además, se hace necesario llevar a los educandos a realizar una lectura y escritura espontánea y por lo tanto comprensible, lo que permitirá expresar con sus propias palabras y desde su punto de vista dar una opinión acerca de lo leído.

Asimismo, las estrategias didácticas pueden concebirse como procedimientos $\mathrm{o}$ arreglos utilizados por el docente en forma flexible y estratégica, para promover la mayor cantidad y calidad de aprendizajes significativos en los estudiantes, por lo que es necesario que el profesor domine las secuencias de acciones e incluso sus técnicas, además de requerir conocimientos sobre cómo y cuándo aplicarlas flexiblemente.

Por esta razón, esta investigación se propone generar orientaciones teóricas sobre estrategias didácticas para mejorar la lectoescritura en los estudiantes de la escuela Mercedes Díaz de la parroquia Sabana de Mendoza, municipio Sucre, estado Trujillo, en busca de mejorar los procesos de aprendizaje de la lectura y escritura, siendo considerados esenciales para la 
construcción de significados en la escuela, así como para su aprendizaje permanente. Del mismo modo, en la actualidad se habla de fomentar los procesos de lectura y escritura, debido a que es importante para el desarrollo de la comunicación y desarrollar su capacidad creativa que le permita apreciar la lectura y la expresión oral y escrita.

De allí que, una de las posibles causas que pudiera estar afectando los procesos de lectura y escritura, además de su creatividad en el afianzamiento de los mismos, es la falta de preparación del docente y representante para gestionar una acción constructiva en el hogar y el uso de estrategias para el proceso de inicio de lectura y escritura. Con relación a la lectura, esta es una actividad que precisa tanto de la comprensión de lo que dice el texto, como de la capacidad de atención, concentración e interés que tenga el estudiante, que pueda entrar en contacto con la información; y es a través de lo que está escrito, que se logra el proceso de interacción social.

En torno a la escritura al igual que la lectura, el aprendizaje se logra de la misma manera, de hecho, el ser humano se enfrenta diariamente en la casa y en la calle con textos escritos que en algún momento le llamaron la atención y tratará de reproducir formas de escritura. Es por ello, que en una intervención para generar procesos constructivos de la lengua escrita, se debe tener en cuenta lo que el niño ha aprendido previamente. Es decir, descubrir lo que ya sabe para que les brinde oportunidades adecuadas a sus intereses, y de esta manera descubra que las escrituras que están a su alrededor expresan un mensaje y tienen un significado.

\section{Teorías Base que Permean la Investigación}

Las teorías constructivistas, conciben el aprendizaje como un proceso significativo centrado en el alumno y su actividad, contrapuesto al aprendizaje mecanicista o por memorización. Estas teorías, consideran el conocimiento como proceso emprendedor, participativo el cual se va construyendo con las 
relaciones del individuo con su medio, creando gradualmente nuevas experiencias. A continuación, se hará referencia a los aportes teóricos de Lev Vygotsky (1993a), Piaget (1978a) y Emilia Ferreiro (2000a).

\subsection{Enfoque Sociohistórico-Cultural de Lev Vygotsky}

El enfoque de los procesos de desarrollo psicológicos del ser humano, de Lev Vygotsky (1993b), resalta "la importancia de los factores históricos, sociales y culturales del conocimiento, enfatizando en la influencia que han tenido estos aspectos en el desarrollo del individuo" (pág. 113). En otras palabras, se entiende que esta teoría considera que los procesos psicológicos del ser humano dependen de la influencia del entorno en el cual se desenvuelve, incidiendo en el desarrollo del aprendizaje.

Para este autor, la mejor enseñanza y el aprendizaje más significativo del hombre, proviene del propio contexto en donde hace su vida social. De allí que, para conocer el desarrollo, es necesario comprobar primero el nivel de información que trae de situación específica que se quiere indagar. Es decir, el conocimiento adquirido previamente, para luego con la indagación intencional del docente, elevarle el nivel cognitivo haciéndolo más complejo.

En este sentido, Vygotsky (1993c), afirma que "el desarrollo cognoscitivo depende en gran medida de las relaciones de las personas que están presente en el mundo del ser humano y de las herramientas que la cultura le da para apoyar su pensamiento" (pág. 121). De esta manera, se adquiere el conocimiento, ideas, actitudes y valores a través de las relaciones interpersonales que se sostiene con la familia y otros miembros importantes para su vida, se apropian de forma de vivir, actuar y hasta formas de pensar que la cultura familiar le ofrece.

En este sentido, la teoría sociocultural Vigotskiana, destaca la función que desempeña en el desarrollo del individuo, según Vygotsky, (1993d), "los

diálogos cooperativos entre ellos y los miembros de la sociedad con la cual 
comparte; y es a través de estos intercambios que esquematizan la cultura de su comunidad: formas de pensar, de comportarse, costumbres y modo de vida" (pág. 128).

\subsubsection{Enfoque Psicogenético de Jean Piaget}

Jean Piaget (1978b), en su enfoque Psicogenético postula el funcionamiento y contenido mental en el sujeto. Al respeto, Coll, (1995), quien afirma que "la postura constructivista en la educación se alimenta de aportaciones de diversas corrientes psicológicas: el enfoque psicogenético piagetiano, la teoría de esquemas cognitivos, teoría ausubeliana de la asimilación y aprendizaje significativo, psicología sociocultural Vygotskyana, así como algunas teorías instruccionales" (pág. 153). Es decir, en este caso el autor selecciona varios postulados del constructivismo del acumulado de elucidaciones que ofrecen diferentes teorías, tomando aspectos o fragmentos de ellas que teóricamente ayudan a analizar, entender y manifestar los procesos de enseñanza y aprendizaje

De hecho, Piaget (1978c) "desarrolló un modelo explicativo y metodológico sui géneris para demostrar cómo se produce la génesis y evolución de las formas de organización del conocimiento, situándose sobre todo en el interior del sujeto epistémico" (pág. 9). En otras palabras, imposible obviar la huella del pensamiento piagetiano en el contexto educacional, cuando va dirigido al aprendizaje del alumno de forma activa y emancipada tomando en cuenta las capacidades cognoscitivas de los estudiantes.

Sin embargo, se hacen críticas fuertes porque su teoría fue normal en cuanto a lo epistémico y no se detuvo en reflexionar acerca de los procesos de conocimiento referentes a la lectura y escritura. De ahí que, Ferreiro, (2000b), realiza investigaciones de esto a partir de la Teoría Piagetiana.

Según, Ferreiro (2000c) citado en Álvarez y Hasbun, (2014a), "cuando se habla de construcción de la escritura... tampoco se trata de una 
construcción inicial que luego se convierte en una simple suma lineal, como si se tratara de adicionar contenido a lo ya construido" (pág. 29). El primer constructo lineal es el aprestamiento que se consideraba un aspecto esencial para la adquisición de la lectura, la escritura, la que se concebía como un proceso cognitivo al cual se subordinan los componentes neuro-psicomotrices.

Como segundo obstáculo, la línea "estructuralista" el cual significa que, al intentar trasladar los conocimientos de la teoría psicogenética piagetiana al campo de la lengua escrita, se ignoró parcialmente que esta teoría hacía referencia a lo fisicomatemático, y no se tuvieron en cuenta los aspectos de la didáctica de la lengua escrita. Y por último como tercer obstáculo el conductismo el cual planteaba la idea de un control externo de los aprendizajes, pero de esta manera se estaban bloqueando las competencias del sujeto para un aprendizaje autónomo. De lo dicho anteriormente, Ferreiro, (2000d) logró avanzar frente a estos obstáculos y expresa:

...Desde esta teoría (psicogenética) se considera que el niño dispone de una precoz capacidad para leer y escribir; también se considera que el niño vive en un mundo alfabetizado y en consecuencia, elabora ideas propias al respecto y formula diversas hipótesis acerca del sistema de escritura... (pág. 79).

Desde otro punto de vista, Bandura, (1987), citado en Vielma y Salas, (2000), en su interpretación del desarrollo de la teoría psicogenética de Piaget concentró todo su esfuerzo "en la conceptualización, de cómo se desarrolla la mediación y la transformación de las percepciones simples convertidos en modelos imitables" (pág. 35). En este orden aclaratorio, acentuó en el rol del tipo adulto en la transferencia social, situación que fue vista como un gran logro dentro de su línea de investigación, ya que con la concepción de la socialización a través de modelos se plantea una nueva forma de explicar las condicionantes del desarrollo durante la niñez. En definitiva, la lectura y escritura en su deber ser está en sitio de honor en los contextos educativos, 
solicitando ayuda de docentes y representantes porque se hace necesario reconocer que el aprendizaje de la lengua escrita es complejo y sistemático.

\subsection{Estrategias Didácticas para Mejorar la Lecto-Escritura}

Las estrategias didácticas, según Tenutto y otros (2011), son "una forma de encarar las prácticas de aula enfrentando sus problemas y buscando los mejores caminos para resolverlos" (pág. 769). Se puede interpretar la definición anterior, como formas específicas de organizar los recursos para obtener resultados consistentes al llevar a cabo el proceso educativo.

Dentro de ese contexto, Camilloni, (2011), define las estrategias didácticas como "las formas en que el docente crea una situación que permita al alumno desarrollar la actividad de aprendizaje, recomendándose planificar una para cada situación didáctica concreta y su variedad depende de la creatividad del profesor" (pág. 32).

Al respecto, se menciona a Maldonado, (2010), que propone un conjunto de orientaciones teóricas para elaborarlas: a) seleccionar aquella que promueve de forma efectiva el aprendizaje de los (as) estudiantes; b) considerar la filosofía educativa prevaleciente; c) conocer a los estudiantes, en términos de sus experiencias, habilidades y estilos de aprendizaje. Asimismo, recomienda: d) identificar su estilo de enseñanza; e) conocer y dominar los diversos tipos de estrategias; f) arriesgarse a experimentar actividades innovadoras y retadoras; g) evaluar los recursos con los que cuenta para apoyar sus iniciativas; (e) seleccionar cuáles estrategias son más apropiadas para cumplir con los objetivos de la clase.

Además, las estrategias didácticas se manifiestan a través de las orientaciones teóricas que el profesor induce en el aula, explicaciones dadas a sus alumnos, así como en la preparación de materiales de apoyo y enseñanza. Al respecto, Orantes (2010), las define como: 
...aquellas que definen las dimensiones del espacio de interacción entre el componente que enseña y el que aprende, identificando los tipos de control dirigidos a facilitar el aprendizaje. Estas dimensiones reflejan factores identificados, por lo general, en forma empírica. El docente actúa dentro de estas dimensiones, de acuerdo con un conjunto de lineamientos que orientan sus acciones para ejercer influencia sobre los diferentes factores que, a su entender, afectan el proceso de enseñanza (pág. 336).

En otras palabras, las estrategias didácticas manifiestan la relación entre las concepciones, supuestos, metodologías que posee el profesor sobre el proceso de enseñanza, en un momento dado de su desarrollo profesional. En concordancia con lo antes expuesto, Mackeer (2008), las conceptualiza como "aquellas acciones que realiza el profesor con el propósito de facilitar la formación y el aprendizaje de las disciplinas en los estudiantes" (pág. 13).

De acuerdo con lo antes señalado, las estrategias didácticas facilitan que el docente provea en sus estudiantes procesos intelectuales simples y complejos, así como desarrollándose no sólo en un dominio de conocimiento específico, sino en situaciones similares gracias al fomento de habilidades de transferencia, favoreciendo con ello su saber cognitivo. De manera, concluyente, mediante su uso es posible organizar, agrupar o clasificar los conceptos con la intención de lograr su representación correcta, explotando ya sea las posibles relaciones entre sus distintas partes y/o con las formas de organización esquemáticas internalizadas.

Referente, a ser usadas como orientaciones teóricas Jolibert, (2001), aporta "una propuesta didáctica integrada, globalizante, que abarca tanto el aprender a leer como el aprender a producir textos en la escuela" (pág. 91). Se plantea como punto de partida que aprender a leer es aprender a interrogar textos completos, desde la educación inicial; el segundo principio en el que se basa sostiene que interrogar un texto implica construir activamente un significado, en función de sus necesidades y sus proyectos, a partir de 
diferentes claves, de distinta naturaleza y de estrategias pertinentes para articularlas.

El tercero de los principios expresa que todo aprendizaje consiste en pasar de una complejidad percibida como borrosa a una complejidad estructurada, poco a poco, por una larga práctica de interacción con ella; un cuarto sostiene que no se puede separar el aprender a leer del aprender a producir; se aprende a leer produciendo textos y se aprende a producir textos leyendo; un quinto principio destaca que se pueden demarcar competencias lingüísticas comunes utilizadas tanto para aprender a leer como para aprender a producir textos.

En definitiva, esta autora revaloriza la enseñanza, con énfasis en que el objetivo no es enseñar a leer, sino formar niños lectores. Con este recuento de propuestas, se ha querido ejemplificar de qué manera se puede realizar la aproximación al texto escrito, tanto desde el punto de vista de la escritura como de la lectura, con el propósito de desmitificar y argumentar la pertinencia de introducir la lengua escrita en la educación inicial.

\subsection{Enseñanza de la Lectura}

La lectura y la escritura son procesos complejos de comprensión y producción de textos escritos, y permiten al estudiante construir aprendizajes en todas las áreas académicas, el acceso al conocimiento reconstruido, así como manifestar sus ideas, opiniones, saberes previos, incrementando de esta manera su bagaje intelectual. De acuerdo con Ortiz, (2008), se entiende la lectura como:

La lectura debe realizarse desde los más tempranos niveles de la enseñanza, esto es, aún desde que los alumnos no pueden hacerlo por sí mismos. Por tal motivo, la lectura diaria de textos interesantes para los niños, los ayuda a percibirse como lectores y le permite ampliar sus conocimientos. Se leerán no solamente los materiales seleccionados para trabajar de la manera antes indicada, sino también otros que le permitan 
entrar en contacto con la literatura y jugar con el lenguaje literario creando y recreando: versos, poemas, rimas, coplas, retahílas, adivinanzas, refranes, cuentos, entre otros (pág. 49).

Cuando se juega con el lenguaje se sitúan en marcha los mecanismos psíquicos de la imaginación y la creatividad, lo que se constituye en motor de crecimiento mental, físico y espiritual. Por ello, compartir la lectura como estrategia didáctica se tiene que consiste en: leer a los niños, leer con los niños y leer para los niños. La primera, leer a los niños sucede cuando se observa que los niños/as no están en capacidad de realizar lecturas por si solos, y cuando ellos lo soliciten, así ya estén alfabetizados.

Entretanto, la segunda leer con los niños, consiste en acompañarlos durante la lectura de manera individual, respondiendo a sus solicitudes, ya sean estas interrogantes, dudas acerca de la interpretación del texto o simplemente cuando pidan ayuda para concluir la lectura. Por último, leer para los niños, tiene como finalidad hacer partícipes a los estudiantes de un texto que el adulto significativo disfrutó y sintió la necesidad de compartirlo. Generalmente este tipo de lectura es realizado en voz alta.

La lectura como se ha señalado anteriormente, puede comenzar mucho antes que en la etapa escolar. Una vez que el niño alcanza ésta, se sugieren actividades que apoyen la transición a la palabra escrita. Esto se logra al fomentar que los niños usen estrategias como, por ejemplo, "la confirmación, la autocorrección, la predicción, la inferencia y la voluntad de arriesgarse". Esto se logra mediante el uso de materiales auténticos, no de programas de libros de texto.

\subsection{Enseñanza de la Escritura}

En relación con la escritura, ésta puede concebirse como un acto de expresión, el cual constituye no solamente un instrumento de comunicación, sino esencialmente una herramienta que permite al individuo reflexionar sobre 
su pensamiento, reorganizando y produciendo conocimientos, ideas, pensamientos nuevos.

Desde esa perspectiva, Flower y Hayes (2009), expresan que, "de acuerdo con los estudios de la génesis textual, la escritura constituye un proceso laborioso, el cual involucra tres grandes tipos de operaciones intervinientes de forma recursiva y variable, según los sujetos escritores, así como el tipo de texto" (pág. 33). Estas operaciones se interrelacionan durante el acto de escritura, en ningún momento constituyen etapas lineales.

Así, el acto de leer y escribir debe avanzar unido uno del otro. Según, Ferreiro (2000e), citado en Álvarez y Hasbun, (2014b), "los estudiantes antes de llevar a cabo una lectura convencional, logran una interpretación de textos libros, cuentos, publicidad, recetas, y ésta reside en darle sentido a los escritos... a través de imágenes, símbolos, todo esto desde interpretación basada en hipótesis personales" (pág. 21).

De esta manera, la autora expresa que el aprendizaje de la escritura se desarrolla paralela y paulatinamente con la lectura. Así pues, se le da al sujeto la oportunidad de escribir cartas, mensajes, reacciones a lecturas, cuentos, entre otros. Los trabajos escritos pueden ser guardados en portafolios en los que se evaluará el proceso por el que ha pasado el niño en la creación de sus escritos.

Es decir, se enfatiza la escritura como proceso, no como producto, guiando al niño en las fases más importantes de la misma a saber: selección del tópico, expresión de ideas, revisión y publicación. El sujeto puede ser ayudado en este proceso por el maestro o la maestra o por sus compañeros y se le brinda oportunidad de compartir sus escritos en diversos medios de publicación. El maestro o la maestra no enfatizan la corrección ortográfica, sino la expresión del significado dando prioridad a la función sobre la forma. En síntesis, para la presentación de esta revisión teórica se han comentado la enseñanza de la escritura y la lectura de forma separada, ambas áreas son 
vistas por los educadores integrales como integrados e indivisibles y de esa forma son tratadas en el salón de clases. 
2.- Ejecución de la Investigación: Se procedió a seleccionar las fuentes de información, organizando los datos provenientes de texto, archivos, computador. Es decir, una vez teniendo un esquema de conceptos definitivo, se procede a desplegar los sitios indicados en el bosquejo. Para esto, se realiza el proceso cognitivo del análisis de los documentos, sintetizando los compendios más característicos, es decir aquéllos que respondan a los objetivos trazados. Además, el investigador contribuye con su interpretación a darle un diferente matiz a la indagación.

3.- Formulación y Presentación de la Información: al tener claro nuestro objetivo generar orientaciones teóricas sobre estrategias didácticas para mejorar la lectoescritura en los estudiantes de la escuela Mercedes Díaz de la parroquia Sabana de Mendoza, municipio Sucre, estado Trujillo, se inició la redacción final del artículo/ensayo, quedando definido tal como se muestra. No obstante, se acota que, aunque fueron otros quienes produjeron inicialmente gran parte de la búsqueda, como investigadores documentales se vivió una práctica de exploración análoga a las que vivieron los antecesores, donde se analizó, sintetizó e interpretaron informaciones para convertirlas en conocimientos.

\section{Conclusiones}

Se establecieron una serie de elementos, enfatizando la construcción de conocimientos, aprendizaje permanente, juicio crítico. Se asumió que el aprendizaje de la lectoescritura posibilita que el (la) estudiante alcance efectivamente estos elementos. La percepción dio como resultado que el niño (a) aprende la lectoescritura porque le permite acceder a conocimientos más complejos, pero si las estrategias didácticas utilizadas por el docente no son las más adecuadas para propiciar la comprensión lectora, que es la finalidad de la lectura, su aprendizaje sólo alcanzará el nivel literal de la lectura, esto es 
la decodificación, con el cual sólo reconocerá grafemas y morfemas, pero sin obtener el significado del texto leído.

Se plantean orientaciones teóricas sobre estrategias didácticas para mejorar la lectoescritura en los estudiantes de la escuela Mercedes Díaz de la parroquia Sabana de Mendoza, municipio Sucre, estado Trujillo, las cuales se generaron con base a lo expuesto previamente, logrando construir una serie de aspectos que posibiliten un mejoramiento de la lectoescritura en los estudiantes de esa institución, siendo válidos para las demás instituciones educativas.

\section{Referencias}

Alfonzo, I. (1994). Técnicas de Investigación Bibliográfica. Caracas: Contexto Ediciones.

Álvarez, D. y Hasbun, V. (2014a,b). Alfabetización Inicial desde el Enfoque Psicogenético. Tesis para Optar al Título de Profesora en Educación Diferencial con Mención en Dificultades

Blaxter, L. Hughes, C. y Tight, M. (2003). Cómo se hace una investigación. España: Gedisa. pp. 193-233.

Camilloni, N. (2011). "El arte de enseñar". Editorial Homosapiens. Buenos Aires.

Coll, C. (1995). La psicología de l'educació: una disciplina aplicada. En C. Coll (Coord.), Psicología de l'educació. Barcelona: Universitat Oberta de Catalunya.

Ferreiro E. (2000a,b,c,d,e). Cultura escrita y educación. México. D.F.: Fondo de Cultura Económica.

Flower y Hayes (2009). La teoría de la redacción como proceso cognitivo. Editorial Cátedra. MadMackeer, T. (2008). "Didáctica”. Editorial Síntesis. Madrid. 
Jolibert, J. (2001). Formar lectores/productores de textos. Propuesta de una problemática didáctica integrada. En: Bofarull et al., Comprensión lectora. El uso de la lengua como procedimiento. (pp. 7995). Barcelona: Graó.

Maldonado, A. (2010). "Problemas de lectura y metodología del aprendizaje". Editorial Escuela Española. Madrid.

Morales, O. (2003). Fundamentos de la Investigación Documental y la Monografía. En Manual para la elaboración y presentación de la monografía. (Norelkys Espinoza y Ángel Rincón, Editores). Mérida, Venezuela: Grupo Multidisciplinario de Investigación en Odontología, Facultad de Odontología. Universidad de Los Andes.

Nogales, (2000a,b). Estrategias Didácticas. Editores Castellos. Caracas, Venezuela.

Orantes, R. (2010). Pedagogía y Didáctica. Editorial Narcea. Madrid.

Ortiz, B. (2008). El aprendizaje y la enseñanza de la lectura y la escritura. Colección Procesos Educativos. Fe y Alegría. Caracas.

Palella, S. y Martins, F. (2010). Metodología de la investigación cuantitativa. 2da. Edición. FEDEUPEL.

Piaget, J. (1978a,b,c). El Desarrollo Cognitivo del Niño. México: Progreso.

Tenutto, F. y otros (2011). "Didáctica”. Editorial Síntesis. Madrid.

Vielma, E. y Salas, M. (2000). Aportes de las teorías de Vygotsky, Piaget, Bandura y Bruner. Paralelismo en sus posiciones en relación con el desarrollo. Educere, vol. 3, núm. 9, junio, 2000, pp. 30-37 Universidad de los Andes, Mérida, Venezuela.

Vygotsky, L. (1993a,b,c,d). Connotaciones y aplicaciones de la psicología sociohistórica en la Educación. Editorial Aiqué. 


\section{Elide del Rosario Castellanos Santiago \\ e-mail: elidecastellanos255@hotmail.com}

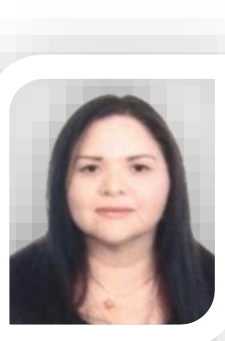

Nacida en Valera. Estado Trujillo, Venezuela. En este momento, curso estudios de: Doctorado en Educación en la Universidad Nacional Experimental Rafael María Baralt en Cabimas- Estado Zulia. Venezuela. Cursé la Licenciatura en Educación Integral en La U.N.E.R.M.B. Título Obtenido: Licenciada en Educación Integral. Fecha: 18 de marzo de 2005. La Maestría en Docencia para Educación Superior en La U.N.ER.M.B. Título Obtenido: Magister Scientiarum en Docencia para Educación Superior. Fecha: 02 de diciembre de 2010.

Mi desempeño actualmente es como docente en el Ministerio del Poder Popular para la Educación. N.E.R. 233 desde 16 de septiembre 2005 hasta la presente y en la Misión Sucre Aldea Universitaria "Cruz Carrillo" Como: Profesora Asesora del Programa Nacional de Gestión Social. En Sabana de Mendoza desde septiembre del 2008 hasta la presente fecha (2017). 


\section{Javier José Castro Capitillo}

e-mail: jccapitillo@hotmail.com

Nací en Cabimas, Estado Zulia, Venezuela. Cursé

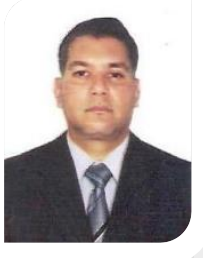
estudios en: Universidad Nacional Experimental "Rafael María Baralt" Título obtenido: Licenciado en Administración, Mención Gerencia Industrial. Fecha: Octubre 1999, Cabimas, Estado Zulia, Venezuela. Universidad Nacional Experimental "Rafael María Baralt" Título obtenido: Magister Scientiarum en Gerencia de Recursos Humanos. Fecha: Mayo 2010, Maracaibo, Estado Zulia, Venezuela.

Universidad Nacional Experimental "Rafael María Baralt" En curso: Doctorado en Educación. Fecha: Febrero 2017, Cabimas, Estado Zulia, Venezuela. Universidad Nacional Experimental "Rafael María Baralt" Título obtenido: "Formación Docente para Egresados". (Componente Docente). Fecha: septiembre 2006-2007, Cabimas, Estado Zulia, Venezuela.

Actualmente me desempeño en la Universidad Nacional Experimental "Rafael María Baralt, Sede Mene Grande, Mene Grande Municipio Baralt, Edo- Zulia. Cargo: Docente de las materias: Gerencia de Mercado, Taller de Acción Profesional I y II, Matemáticas II, Estrategias Gerencial. Finanzas de la Empresa. (Programa de Administración). Fundamentos Matemáticos. Aritmética I (Programa de Educación) Períodos: Abril 2005, Período actual (2017).

El contenido de este manuscrito se difunde bajo una Licencia de Creative Commons ReconocimientoNoComercial-Compartirlgual 4.0 Internacional 